\title{
Concept Extraction and Prerequisite Relation Learning from Educational Data
}

\author{
Weiming Lu, Yangfan Zhou, Jiale Yu, Chenhao Jia \\ College of Computer Science and Technology, Zhejiang University, Hangzhou, China \\ \{luwm, 21621119, 21721115, 21821117\}@zju.edu.cn
}

\begin{abstract}
Prerequisite relations among concepts are crucial for educational applications. However, it is difficult to automatically extract domain-specific concepts and learn the prerequisite relations among them without labeled data.

In this paper, we first extract high-quality phrases from a set of educational data, and identify the domain-specific concepts by a graph based ranking method. Then, we propose an iterative prerequisite relation learning framework, called iPRL, which combines a learning based model and recovery based model to leverage both concept pair features and dependencies among learning materials. In experiments, we evaluated our approach on two real-world datasets Textbook Dataset and MOOC Dataset, and validated that our approach can achieve better performance than existing methods. Finally, we also illustrate some examples of our approach.
\end{abstract}

\section{Introduction}

Domain-specific concepts and their prerequisite relations are the core of knowledge space and personalized learning theory (Falmagne et al. 2006). Prerequisite relations can be essentially considered as the dependency among concepts, and they are crucial for people to learn, organize, apply and generate knowledge (Margolis and Laurence 1999). For example, the prerequisite knowledge of "Conditional Random Fields" is "Hidden Markov Model". Thus, organizing the knowledge in prerequisite relations can improve the educational tasks, such as curriculum planning (Liu et al. 2016) and intelligent tutoring (Wang and Liu 2016).

However, recent researches mainly focus on keyword extraction (Mihalcea and Tarau 2004; Liu, Chen, and Sun 2011; Tixier, Malliaros, and Vazirgiannis 2016) or highquality phrase mining (Liu et al. 2015; Shang et al. 2018), and there are only a few efforts aiming to extract domainspecific concepts and learn prerequisite relations from educational data, such as courses (Yang et al. 2015; Liang et al. 2015; Liu et al. 2016; Liang et al. 2017), MOOCs (Massive Open Online Courses) (Pan et al. 2017a), textbooks (Wang et al. 2016; Liang et al. 2018) and scientific papers(Gordon et al. 2016).

The prerequisite relation learning methods can be classified into three categories: local statistical information based

Copyright (C) 2019, Association for the Advancement of Artificial Intelligence (www.aaai.org). All rights reserved. methods, learning based methods and recovery based methods. For the local statistical information, reference distance and cross-entropy were proposed (Liang et al. 2015; Gordon et al. 2016) to measure prerequisite relations among concepts. For the learning based methods, several features were proposed for the prerequisite classification. For example, Pan et al. (2017a) proposed contextual, structural and semantic features, and Liang et al. (2018) utilized graph-based and text-based features. In contrast, recovery based methods do not need to extract features to learn a prerequisite classifier. For example, Liang et al. (2017) can recover concept prerequisite relations from course dependencies directly.

However, there are still many challenges to automatically mine prerequisite relations among domain-specific concepts. (1) It is difficult to extract fine-grained concepts for each domain. (2) It is time-consuming to label the prerequisite relations in the learning based methods. (3) The recovery based methods only rely on the dependency among educational data, but neglect the contextual, structural and semantic features.

In order to address these challenges, we propose a domain-specific concept extraction approach, called DsCE, and an iterative prerequisite relation learning approach, called iPRL. DsCE can extract high-quality phrases from documents firstly, and then identifying domain-specific concepts with a graph based ranking method. While iPRL can utilize both the advantage of the learning based model and the recovery based model. It can learn the prerequisite relations among concepts without human labeled data, and improve the performance gradually with the interaction between two models.

We conduct extensive experiments on two real-world datasets: Textbook and MOOC datasets. The results show that our approach outperforms the state-of-the-art methods.

\section{Our Approach}

\section{Problem Formulation}

As shown in Figure 1, given a set of educational data in one domain, which is modeled as a set of sequential learning materials (denoted as LMs for short) such as textbook chapters and MOOC videos, we want to extract the domainspecific concepts and the prerequisite relations among them from these data. 


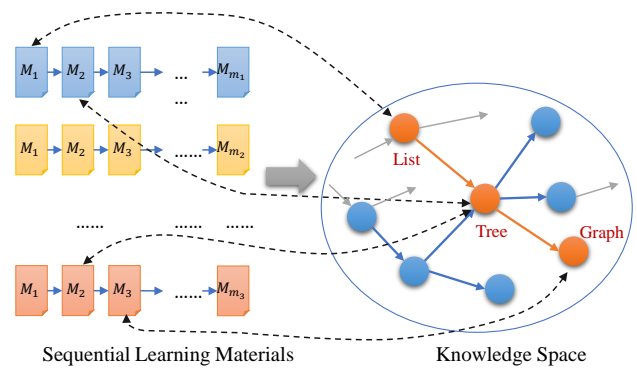

Figure 1: Illustration of Domain-specific Concept Extraction and Prerequisite Relation Learning from Sequential Learning Materials, which are denoted as $\left\{M_{1}, M_{2}, \ldots, M_{m_{i}}\right\}$.

For convenience, we list the main symbols used in this paper in Table 1.

\begin{tabular}{|c|c|}
\hline Symbol & Meaning \\
\hline $\mathcal{D}$ & $\begin{array}{l}\text { a set of educational data in one domain, and } \mathcal{D}= \\
\left\{d_{m}\right\}^{|\mathcal{D}|}\end{array}$ \\
\hline $\mathcal{C}$ & a set of concepts extracted from $\mathcal{D}$, and $\mathcal{C}=$ \\
\hline $\mathcal{P}$ & $\begin{array}{l}\left\{{ }^{i}\right\}_{i=1} \text { : } \\
\mathcal{P}=\left\{p_{i}\right\}^{|\mathcal{P}|}\end{array}$ \\
\hline$d_{m} \in \mathcal{D}$ & $\begin{array}{l}\text { an educational data with a sequential LMs, such as } \\
\text { a book with a sequence of chapters or a MOOC } \\
\text { course with a sequence of videos. }\end{array}$ \\
\hline $\begin{array}{c}Q\left(p_{i}\right) \\
\operatorname{conf}\left(p_{i}\right)\end{array}$ & $\begin{array}{l}\text { the quality score of } p_{i} \in \mathcal{P} \text { of being a phrase. } \\
\text { the confidence score of } p_{i} \in \mathcal{P} \text { of being a domain- } \\
\text { specific concept. }\end{array}$ \\
\hline $\mathbf{x}_{i}^{m} \in \mathbb{R}^{|\mathcal{C}|}$ & $\begin{array}{l}\text { the vector representation of the } i^{t h} \mathrm{LM} M_{i}^{m} \in d_{m} \\
\text { in concept space } \mathcal{C} \text {. }\end{array}$ \\
\hline$\mapsto$ & prerequisite relation between concepts or LMs. \\
\hline$\Omega_{m}$ & $\begin{array}{l}\text { a set of prerequisite relations among LMs, and } \\
\Omega_{m}=\{i \mapsto j\}_{M_{i}^{m}, M_{j}^{m} \in d_{m}, M_{i}^{m} \mapsto M_{j}^{m}, d_{m} \in \mathcal{D}} \text {. }\end{array}$ \\
\hline A & $\begin{array}{l}\mathbf{A}=\left(a_{i, j}\right) \in[-1,1]^{|\mathcal{C}| \times|\mathcal{C}|} \text { represents prerequisite } \\
\text { relations among concepts which is obtained from a } \\
\text { recovery based model, and } a_{i, j} \text { is the weight quan- } \\
\text { tifying how concept } i \text { depends on concept } j \text {. }\end{array}$ \\
\hline $\mathbf{F}$ & $\begin{array}{l}\mathbf{F}=\left(f_{i, j}\right) \in[-1,1]^{|\mathcal{C}| \times|\mathcal{C}|} \text { represents the pre- } \\
\text { dicted results from a learning based model } \mathcal{F} \text {, and } \\
f_{i, j} \text { is the predicted result for concept } i \text { and concept } \\
j .\end{array}$ \\
\hline W & $\begin{array}{l}\mathbf{W}=\left(w_{i, j}\right) \in[0,1]^{|\mathcal{C}| \times|\mathcal{C}|} \text { represents the related- } \\
\text { ness among concepts. }\end{array}$ \\
\hline
\end{tabular}

Table 1: Meaning of symbols used

Therefore, the problem could be formally defined as: given a set of educational data in one domain, $\mathcal{D}=$ $\left\{d_{m}\right\}_{m=1}^{M}$, and each data is a sequential learning materials $d_{m}=\left\{M_{i}^{m}\right\}_{i=1}^{\left|d_{m}\right|}$. The goal is to extract domain-specific concepts $\mathcal{C}$ from $\mathcal{D}$, and then learn the prerequisite relation matrix $\mathbf{A}$ among these concepts by an iterative prerequisite relation learning approach.

\section{Domain-specific Concept Extraction}

Domain-specific concepts should be identified before prerequisite relation learning.

However, it is time consuming and tedious to annotate all fine-grained concepts in each domain, and pre- defined part-of-speech rules such as Noun $^{+}$Noun and $[\text { Adj } \mid \text { Noun }]^{+}$Noun are unsuitable for the extraction task. Because some domain-specific concepts are complex, e.g., non-homogeneous linear differential equation, and linear differential equation with constant coefficients of the second order in the Calculus domain. In addition, not all phrases are domain-specific, e.g., basic theory is a good phrase, but it is not a domain-specific concept.

We developed an unsupervised and domain-independent approach (DsCE) to extract domain-specific concepts from textbooks, which consists of two steps: (1) extracting highquality phrases from textbooks. (2) identifying domainspecific concepts from the phrases.

Extracting high-quality phrases A phrase is defined as a sequence of words that appear consecutively in the text, forming a complete semantic unit in certain contexts of the given documents (Finch 2000). A high-quality phrase is a phrase which has a high probability to be a complete semantic unit, meeting the criteria of Popularity, Concordance, Informativeness and Completeness (Liu et al. 2015; Shang et al. 2018).

Given the set of educational data $\mathcal{D}$ in one domain, we used AutoPhrase (Shang et al. 2018) to extract the highquality phrases $\mathcal{P}$ to form the candidates of domain-specific concepts, since AutoPhrase has the following advantages: (1) It is a robust positive-only distant training method, which can be used in many different domains with the minimal human effort. (2) It can leverage part-of-speech (POS) tags to further enhance the extraction performance.

However, the high-quality phrases extracted by $A u$ toPhrase may not be domain-specific. We extracted phrases from textbooks in three domains: "Calculus"(CAL), "Data Structure"(DS), and "Physics"(PHY), and calculated the average ratio of the domain-specific concepts in top K phrases according to its quality score. The result is shown in Figure 2 , which demonstrates that high-quality phrases may not be domain-specific, and has many noise in the top K phrases. Therefore, we should identify the domain-specific concepts from these high-quality phrases.

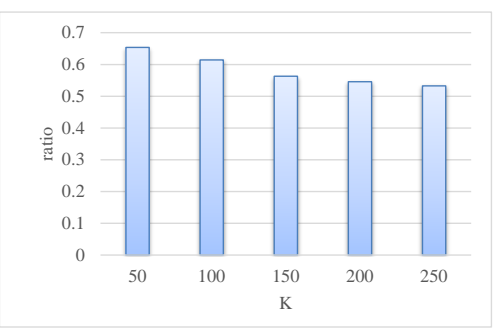

Figure 2: The ratio of the domain-specific concepts in top K phrases of the results of AutoPhrase.

Identifying domain-specific concepts In order to identify domain-specific concepts in the high-quality phrases, we resorted to a graph propagation based ranking method (Pan et al. 2017b).

First, a weighted undirected fully-connected graph $\mathcal{G}=$ $(V, E)$ was constructed, where $V$ is the vertex set of $\mathcal{G}$ and 
$E$ is the edge set of $\mathcal{G}$. Each vertex in $V$ is a phrase $p_{i} \in \mathcal{P}$ with a quality score $Q\left(p_{i}\right)$ extracted by AutoPhrase. For each edge $\left(p_{i}, p_{j}\right) \in E$, its weight $w\left(p_{i}, p_{j}\right)$ is the semantic relatedness of the two phrases $p_{i}$ and $p_{j}$. We trained word embedding vectors based an online encyclopedia corpus via word2vec (Mikolov et al. 2013b), and then obtained the semantic representation of each phrase via the vector addition of its individual word vectors. Then, the semantic relatedness of two phrases is defined as the cosine similarity of their vectors.

Second, a propagation based ranking method was executed on the graph $\mathcal{G}$ with the assumption that highconfidence concepts in the graph could propagate their confidence scores to their neighbor nodes that have high semantic relatedness with them, to discover other potential domain-specific concepts. Each vertex $p_{i}$ has a confidence score $\operatorname{conf}\left(p_{i}\right)$ of being a domain-specific concept, and con $f^{k}\left(p_{i}\right)$ is the score of $p_{i}$ in the $k$-th iteration of the propagation. We set the initial confidence score of each vertex as $\operatorname{con} f^{0}\left(p_{i}\right)=1$. The propagation functions are defined as:

$$
\begin{aligned}
s^{k}\left(p_{j}, p_{i}\right) & =\operatorname{opf}\left(p_{i}, p_{j}\right) \cdot Q\left(p_{j}\right) \cdot e\left(p_{i}, p_{j}\right) \cdot \operatorname{conf}^{k}\left(p_{j}\right) \\
\operatorname{conf} f^{k+1}\left(p_{i}\right) & =\frac{1}{Z}\left(\frac{\sum_{p_{j} \in A\left(p_{i}\right)} s^{k}\left(p_{j}, p_{i}\right)}{\left|A\left(p_{i}\right)\right|}\right)
\end{aligned}
$$

where $s^{k}\left(p_{j}, p_{i}\right)$ is the voting score that $p_{j}$ propagates to $p_{i}$ in the $k$-th iteration, which is determined by the semantic relatedness between $p_{i}$ and $p_{j} e\left(p_{i}, p_{j}\right)$, the quality score of $p_{j} Q\left(p_{j}\right)$, the confidence score of $p_{j}$ in the $k$-th iteration $\operatorname{con} f^{k}\left(p_{j}\right)$, and the overlapping penalty between $p_{i}$ and $p_{j}$ opf $\left(p_{i}, p_{j}\right)$. If $p_{i}$ and $p_{j}$ contain one or more identical words, we say they are overlapping, and should be penalized during the score propagation. For example, this algorithm and sort algorithm are related by cosine similarity, but sort algorithm should not propagate its confidence score to this algorithm, since this algorithm is not a domain-specific concept. We set opf $\left(p_{i}, p_{j}\right)=1$, if $p_{i}$ and $p_{j}$ are not overlapping. Otherwise, $\operatorname{opf}\left(p_{i}, p_{j}\right)=\lambda, \lambda \in(0,1)$ (We set $\lambda=0.5$ in the experiments). con $f^{k+1}\left(p_{i}\right)$ is the new confidence score of $p_{i}$, which is dependent on the average voting score of vertexes in $A\left(p_{i}\right) . A\left(p_{i}\right)$ is the vertex set which will propagate the voting scores to $p_{i}$ in each iteration. After each iteration, the confidence scores should be normalized, so $Z$ is the normalization factor.

Finally, the phrases with the confidence score greater than $\phi=0.6$ are selected as the domain-specific concepts $\mathcal{C}$.

\section{Prerequisite Relation Learning}

After obtained the domain-specific concepts $\mathcal{C}$, we would like to learn the prerequisite relations among these concepts.

Inspired by Liang et al. (2017), we observed that (1) The dependency among learning materials is caused by sufficient evidence provided by prerequisite relations among concepts in that materials. This means if one learning material depends on another one for knowledge learning, there must be sufficient concept pairs between them which have prerequisite relations. Taking Figure 3(a) as an example, because chapter Tree $\mapsto$ Graph, then the concepts in these chapters would like to have prerequisite relations, such as binary tree $\mapsto$ undirected graph and B tree $\mapsto D A G$ graph, where $\mapsto$ means there is a prerequisite relation between LMs or concepts.

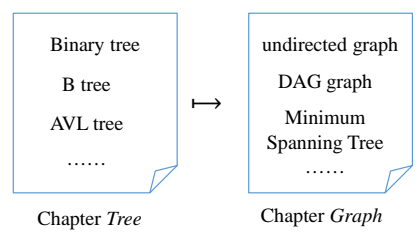

(a) Observation 1

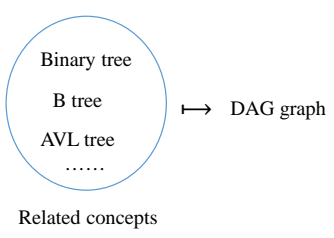

(b) Observation 2

Figure 3: The observations of the prerequisite relations among concepts.

(2) Related concepts would have similar prerequisite relations with other concepts. As in Figure 3(b), binary tree and $B$ tree are highly related, so if binary tree $\mapsto D A G$ graph, then $B$ tree $\mapsto D A G$ graph.

(3) The results of the recovery based model and the learning based model should be consistent. That is, it would be better that $\mathbf{A}$ equals to $\mathbf{F}$, where $\mathbf{A}$ is the result of the recovery based model and $\mathbf{F}$ is the result of the learning based model.

Thus, the optimization function is designed as follows.

$$
\begin{array}{ll}
\min _{\mathbf{A}, \xi, \mathbf{F}} \quad & \|\xi\|_{2}+\lambda_{1} \frac{\|\mathbf{A}-\mathbf{F}\|_{F}}{|\mathcal{C}|^{2}}+ \\
& \lambda_{2} \sum_{1 \leq i \neq j \leq|\mathcal{C}|} w_{i, j} \frac{\|\mathbf{A}(i,:)-\mathbf{A}(j,:)\|_{2}}{|\mathcal{C}|} \\
\text { s.t. } \quad & \left(\mathbf{x}_{i}^{m}\right)^{T}\left[\mathbf{C}_{i \mapsto j}^{m} \odot \mathbf{A}\right] \mathbf{x}_{j}^{m} \geq \theta-\xi_{i, j}^{m}, \\
& \forall d_{m} \in \mathcal{D},(i, j) \in \Omega_{m} \\
& \mathbf{A}_{i, j}+\mathbf{A}_{j, i}=0,0<i \neq j<|\mathcal{C}| \\
& -1 \leq \mathbf{A}_{i, j} \leq 1,0<i \neq j<|\mathcal{C}| \\
& \mathbf{A} \odot(\mathbf{1}-\mathbf{D})=\mathbf{0} \\
& \mathbf{A}_{i, j}>0, \forall d_{m} \in \mathcal{D}, \operatorname{pos}_{m}(i)<\operatorname{pos}_{m}(j)
\end{array}
$$

where $\xi_{i, j}^{m}$ is a slack parameter for a pair of LMs $M_{i}^{m} \mapsto$ $M_{j}^{m}$ in $d_{m} \in \mathcal{D}$ which has a prerequisite relation. $\theta, \lambda_{1}$ and $\lambda_{2}$ are positive hyper parameters, and they are set to $1.0,0.2$ and 0.2 in the experiments respectively.

For the objective function, the first term is the empirical loss as in Liang et al. (2017). The second term is based on the third observation, which makes $\mathbf{A}$ be close to $\mathbf{F}$. The third term is based on the second observation, which means if concept $i$ and $j$ are related, $\mathbf{A}(i,:)$ and $\mathbf{A}(j,:)$ should be similar. Here, $w_{i, j}$ is the relatedness between concept $i$ and $j$, which would be computed in advance. How to calculate $\mathbf{F}$ and $\mathbf{W}$ will be elaborated in the following section.

For the constraints, the first three constraints are learned from Liang et al. (2017). The first constraint is based on the first observation mentioned above, where $\odot$ is elementwise product, and $\mathbf{C}_{i \mapsto j}^{m}$ is used to remove the contribution from the common concepts between $M_{i}^{m}$ and $M_{j}^{m}$, so $\mathbf{C}_{i \mapsto j}^{m}=\left(c_{s, t}^{m}\right) \in\{0,1\}^{|\mathcal{C}| \times|\mathcal{C}|}$, and $c_{s, t}^{m}=0$ if $\mathbf{x}_{i}^{m}[s]>0$ or $\mathbf{x}_{j}^{m}[t]>0$, otherwise $c_{s, t}^{m}=1$, and $\xi_{i, j}^{m}$ is the penalty of this constraint. The second constraint means if concept $i$ 
is a prerequisite of $j$, then $j$ is not a prerequisite of $i$. The third constraint bounds the strength of prerequisite relation in $[-1,1]$.

The fourth constraint assumes that there is no prerequisite relation between two concepts if one concept is not contained in other's encyclopedia article according to Talukdar and Cohen (2012). Here, $\mathbf{1}=\{1\}^{|\mathcal{C}| \times|\mathcal{C}|}$, and $\mathbf{D} \in$ $\{0,1\}^{|\mathcal{C}| \times|\mathcal{C}|}$ is introduced to filter concept pairs. When the article of concept $i(j)$ contains concept $j(i), \mathbf{D}_{i, j}=1$, otherwise $\mathbf{D}_{i, j}=0$. The last constraint specifies that if concept $i$ always occurs before $j$, then $i$ may be the prerequisite concept of $j$, but $j$ must not be the prerequisite concept of $i$. Here, $\operatorname{pos}_{m}(i)$ is the position of concept $i$ in $d_{m}$.

The optimization problem is solved iteratively. If we don't have any labeled data, $\mathbf{F}$ is initialized randomly, otherwise a probabilistic classifier $\mathcal{F}$ is trained to predict $\mathbf{F}$. Then, by fixing $\mathbf{F}$, the problem becomes a quadratic optimization problem, and $\mathbf{A}$ and $\xi$ are solved by MOSEK ${ }^{1}$ software. When obtaining a new $\mathbf{A}$, new training data can be selected to enhance the classifier $\mathcal{F}$. In our experiments, the performance of our model increases gradually and tends to converge.

Specifically, during the $k^{t h}$ iterations, three datasets are formed firstly: $P^{k}=\left\{(i, j) \mid \mathbf{A}_{i, j}>\tau\right\} ; N^{k}=$ $\left\{(i, j)|| \mathbf{A}_{i, j} \mid<\tau\right\} ; O^{k}=\left\{(i, j) \mid \mathbf{A}_{i, j}==0\right\}$. Then, the positive samples is formed by selecting the top $\left(\frac{k}{k+1}\right)^{2}\left|P^{k}\right|$ concept pairs from $P^{k}$. The negative samples also have the same number of the positive samples, but they consists of three parts: $20 \%$ data is randomly selected from $\left\{(j, i) \mid(i, j) \in P^{k}\right\}$, and the remaining $80 \%$ data is randomly selected from $N^{k}$ and $O^{k}$ equally. $\tau=0.6$ in our experiments. The procedure is shown in the Algorithm 1.

\footnotetext{
Input: $\mathcal{D}$ : a set of educational data in a domain.

$\mathcal{C}$ : a set of concepts extracted from $\mathcal{D}$ by DsCE.

$\mathbf{x}$ : the vector representations of all learning materials in $\mathcal{D}$.

Output: A: the prerequisite relations among concepts in $\mathcal{C}$.

Initialize $\mathbf{F}$ randomly, or train a probabilistic classifier $\mathcal{F}$

using labeled data to initialize $\mathbf{F}$;

Calculate the concept relatedness matrix $\mathbf{W}$ according to $\mathcal{D}$;

Obtain A by solving Eq. 1 with MOSEK;

while $A$ is not convergent do

Update training data for $\mathcal{F}$ according to $\mathbf{A}$; (Update the

Learning based Model)

Update $\mathbf{F}$ using $\mathcal{F}$;

Fixing $\mathbf{F}$, obtain new $\mathbf{A}$ by solving Eq. 1; (Update the

end Recovery based Model)
}

Algorithm 1: Iterative Prerequisite Relation Learning.

\section{Concept Features and Relatedness}

To train $\mathcal{F}$, we used several features to capture whether a concept pair has a prerequisite relation.

For MOOCs, in order to compare the method in (Pan et al. 2017a), we still used the same features, including semantic relatedness, video reference distance (refd), sen-

\footnotetext{
${ }^{1}$ http://www.mosek.com
}

tence refd, Wikipedia refd, average position distance, distributional asymmetry distance and complexity level distance.

For textbooks, in addition to the features mentioned above, where we replaced video refd and sentence refd with chapter refd, we also added Wikipedia abstract occurrence and content refd.

chapter refd: For a concept pair $(a, b)$, chapter reference weight $(c r w)$, which is to qualify how $b$ is referred by chapters of $a$, is defined as: $\operatorname{crw}(a, b)=$ $\frac{\sum_{d_{m} \in \mathcal{D}} \sum_{M \in d_{m}} f(a, M) \cdot r(M, b)}{\sum_{d_{m} \in \mathcal{D}} \sum_{M \in d_{m}} f(a, M)}$, where $f(a, M)$ indicates the term frequency of concept $a$ in chapter $M$, and $r(M, b) \in$ $\{0,1\}$ denotes whether concept $b$ appears in chapter $M$. Then, chapter refd of $(a, b)$ is $\operatorname{crw}(b, a)-\operatorname{crw}(a, b)$.

Wikipedia abstract occurrence: For a concept pair $(a, b)$, if concept $a$ occurs in the Wikipedia abstract of concept $b$, Wikipedia abstract occurrence of $(a, b)$ is 1 , otherwise 0 .

Wikipedia content refd: For a concept pair $(a, b)$, the Wikipedia content refd is defined as: $f_{b}(a)-f_{a}(b)$, where $f_{b}(a)$ is the term frequency of concept $a$ in the article of $b$.

To define $\mathbf{W}$, we proposed two relatedness measurements between concept $i$ and $j$.

Semantic Relatedness: $\mathbf{W}_{i, j}$ is defined as the normalized cosine distance between two embedding vectors: $\mathbf{W}_{i, j}^{s}=$ $\frac{1}{2}\left(1+\frac{v_{i} \cdot v_{j}}{\left\|v_{i}\right\| \cdot\left\|v_{j}\right\|}\right)$, where $v_{i}$ is the word embedding vector learned by word2vec (Mikolov et al. 2013a).

Position Relatedness: if two concepts have the similar positions in textbooks or MOOC videos, they would have similar prerequisite relations with other concepts. Thus, $\mathbf{W}_{i, j}$ is defined as: $\mathbf{W}_{i, j}^{p}=\frac{1}{2} \sum_{d_{m} \in \mathcal{D}} \sum_{M \in d_{m}}\left(p_{i}^{M}+p_{j}^{M}\right) \mid p_{i}^{M}-$ $p_{j}^{M} \mid$, where $p_{i}^{M \in d_{m}}=\frac{n(i, M)}{n\left(i, d_{m}\right)}, n(i, M)$ is the term frequency of concept $i$ in the material $M$.

\section{Experiments}

In this section, we will evaluate the domain-specific concept extraction and prerequisite learning respectively.

\section{Evaluation on Domain-specific Concept Extraction}

Datasets In order to evaluate the domain-specific concept extraction, we collected six Chinese textbooks in each domain: "Calculus"(CAL), "Data Structure"(DS), and "Physics"(PHY) from our digital library, and then extracted the content of the textbooks for concept extraction.

Baseline Methods We employed the following three baseline methods to compare with our DsCE method.

TextRank (Mihalcea and Tarau 2004): An unsupervised graph-based ranking model for keyword extraction.

THUCKE (Liu, Chen, and Sun 2011) ${ }^{2}$ : A word trigger method for keyword extraction based on word alignment in statistical machine translation.

AutoPhrase (Shang et al. 2018) ${ }^{3}$ : An automated phrase mining method with POS-guided phrasal segmentation.

\footnotetext{
${ }^{2}$ https://github.com/thunlp/THUCKE

${ }^{3}$ https://github.com/shangjingbo1226/AutoPhrase
} 
Performance comparison and analysis To measure performance, we asked three students who are majoring in the corresponding domain to annotate whether the concepts extracted from each method are domain-specific or not, and then took a majority vote of the annotations to create final domain-specific concept set for each domain. Precision and Recall are used to evaluate the extraction results.

In Figure 4, we show the precision of domain-specific concept extraction in top $\mathrm{K}$ results of different methods in the three domains.

We find that our method (DsCE) outperforms baseline methods across all domains. Specifically, we have the following observations. First, AutoPhrase can extract highquality phrases, but it does not focus on keyword extraction, so the top ranked phrases may not be domain-specific. Second, the precision in Calculus domain is relatively low comparing to other two domains. This is because there are many mathematical formulas in the textbooks of Calculus, which affect the OCR (Optical Character Recognition) quality for textbooks. Third, the graph propagation based ranking method can indeed improve the performance of $\mathrm{Au}$ toPhrase for domain-specific concept extraction.

In addition, we also calculate the average precision and recall for each method across all domains, and show the precision-recall curves in Figure 5. Obviously, we find that our method outperforms baseline methods greatly.

Figure 6 illustrates some examples of the top ranked domain-specific concepts extracted for each domain. We find that (1) TextRank and THUCKE are unable to extract complex concepts, which makes them have low recall. (2) Some top-ranked concepts extracted by AutoPhrase are obscure, such as michelson interferometer and Nicol prism, while the basic core concepts are not ranked in the top.

\section{Evaluation on Prerequisite Relation Learning}

Datasets We evaluated the prerequisite relation learning with the following two different datasets.

Textbook: To the best of our knowledge, these is no public dataset for mining prerequisite relation between concepts in textbooks. We also chose Chinese textbooks in three domains: Calculus, Data Structure, and Physics to create the dataset. The construction procedure is as follows. For each domain, we extracted concepts from textbooks by our DsCE method, and asked three students to select the most related domain concepts. Finally, the prerequisite relation among them were annotated through a majority vote strategy.

MOOC: We used MOOC data mentioned in (Pan et al. 2017a), which has been released ${ }^{4}$.

The statistics of the datasets are listed in Table 2, which lists the number of concepts and the positive pairs in each domain.

Baseline Methods We use the following state-of-the-art methods as baselines:

RefD (reference distance) (Liang et al. 2015): A linkbased metric for measuring the prerequisite relations among concepts.

\footnotetext{
${ }^{4}$ http://keg.cs.tsinghua.edu.cn/jietang/software/acl17prerequisite-relation.rar
}

\begin{tabular}{c|c|c|c}
\hline \multicolumn{4}{c}{ Textbook } \\
\hline dataset & \#books & \#concepts & \#pairs(+) \\
\hline CAL & 6 & 89 & 439 \\
\hline DS & 6 & 90 & 453 \\
\hline PHY & 6 & 139 & 630 \\
\hline \hline \multicolumn{4}{|c}{ MOOC } \\
\hline dataset & \#courses & \#concept & \#pairs(+) \\
\hline ML & 5 & 244 & 1,735 \\
\hline DSA & 8 & 201 & 1,148 \\
\hline
\end{tabular}

Table 2: Statistics of the datasets for evaluating on prerequisite relation learning.

CPR-Recover (Liang et al. 2017): An unsupervised method, which can recover concept prerequisite relations from course dependencies. In our datasets, we used it to recover concept prerequisite relations from book chapter or MOOC video dependencies.

PRinMOOC (Pan et al. 2017a): A supervised method for prerequisite relation learning in MOOC, which proposed contextual, structural and semantic features, and then used random forest to capture the prerequisite relations between concepts.

In the experiments, we trained the English and Chinese word vectors by word2vec (Mikolov et al. 2013a) on English Wikipedia and Baidu Baike ${ }^{5}$ respectively, and the dimensions are both set to 100 .

Performance comparison and analysis We evaluated the methods under several scenarios, including with non-labeled data, with labeled data, different concept relatedness and iterative learning.

(1) Evaluation with non-labeled data. We compared iPRL with RefD and CPR-Recover without any labeled data in both Textbook and MOOC datasets. The results are shown in Table 3.

\begin{tabular}{cc|c|c|c|c|c}
\hline \multirow{2}{*}{ Method } & \multicolumn{3}{|c|}{ Textbook } & \multicolumn{2}{c}{ MOOC } \\
\cline { 3 - 7 } & & CAL & DS & PHY & ML & DSA \\
\hline \multirow{2}{*}{ RefD } & $P$ & 35.49 & 34.78 & 27.96 & 71.70 & 72.69 \\
\cline { 3 - 7 } & $R$ & 57.40 & 44.54 & 50.32 & 30.4 & 37.8 \\
\cline { 3 - 7 } & $F_{1}$ & 43.86 & 39.06 & 35.94 & 42.70 & 49.74 \\
\hline \multirow{3}{*}{ CPR-R } & $P$ & 44.69 & 31.70 & 29.07 & 57.79 & 55.94 \\
\cline { 3 - 7 } & $R$ & 62.41 & 57.68 & 60.89 & 35.6 & 35.8 \\
\cline { 3 - 7 } & $F_{1}$ & 52.09 & 40.91 & 39.35 & 44.06 & 43.66 \\
\hline \multirow{2}{*}{ iPRL } & $P$ & 91.38 & 58.86 & 71.69 & 65.72 & 72.48 \\
\cline { 3 - 7 } & $R$ & 55.58 & 64.80 & 57.85 & 46.4 & 43.2 \\
\cline { 3 - 7 } & $F_{1}$ & $\mathbf{6 9 . 1 2}$ & $\mathbf{6 1 . 6 9}$ & $\mathbf{6 4 . 0 6}$ & $\mathbf{5 4 . 4 0}$ & $\mathbf{5 4 . 1 4}$ \\
\hline
\end{tabular}

Table 3: Comparison with non-labeled data(\%) on Textbook and $M O O C$ datasets

From the tables, we can see that (i) iPRL outperforms other methods under the non-labeled data situation, which achieves $+16.69 \%$ for CPR-Recover and $+18.44 \%$ for RefD with respect to the average $F_{1}$. (ii) CPR-Recover can achieve a comparable recall, but its precision is too low.

\footnotetext{
${ }^{5}$ https://baike.baidu.com/
} 


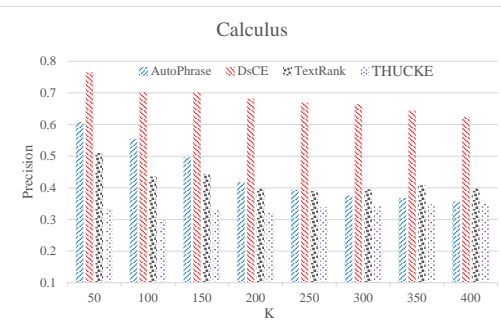

(a) Calculus

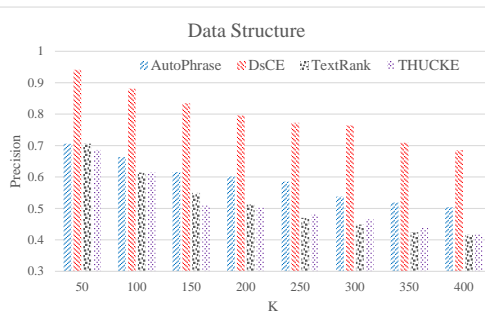

(b) Data Structure

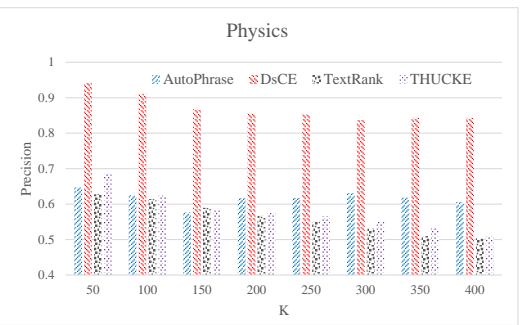

(c) Physics

Figure 4: The precision of domain-specific concept extraction of different methods in three domains.

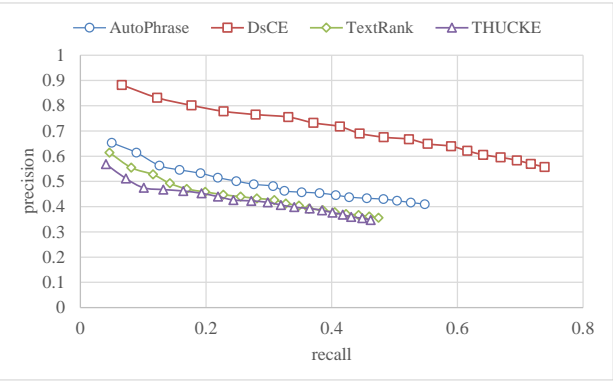

Figure 5: The precision-recall curves of domain-specific concept extraction

(2) Evaluation with labeled data. For the labeled data, we applied 5-fold cross validation to evaluate the performance of the proposed method,i.e., using 4 folds in methods such as PRinMOOC and iPRL, and then testing them with the remaining fold. We also selected random forest as $\mathcal{F}$ as in (Pan et al. 2017a). The comparison between PRinMOOC and iPRL is shown in Table 4.

\begin{tabular}{c|c|c|c}
\hline Method & measure & ML & DSA \\
\hline \multirow{3}{*}{ PRinMOOC } & $P$ & 72.08 & 88.32 \\
\cline { 2 - 4 } & $R$ & 59.4 & 48.4 \\
\cline { 2 - 4 } & $F_{1}$ & 65.13 & 62.53 \\
\hline \multirow{3}{*}{ iPRL } & $P$ & 65.96 & 70.93 \\
\cline { 2 - 4 } & $R$ & 68.6 & 74.2 \\
\cline { 2 - 4 } & $F_{1}$ & $\mathbf{6 7 . 2 5}$ & $\mathbf{7 2 . 5 3}$ \\
\hline
\end{tabular}

Table 4: Comparison with labeled data(\%) on MOOC dataset

From the table, we can see that: (i) iPRL outperforms PRinMOOC, which only has the learning model. (ii) Labeled data can promote iPRL. Comparing Table 4 with Table 3 , the average $F_{1}$ increases by $12.85 \%$ and $18.39 \%$ on ML and DSA datasets.

(3) Evaluation with concept relatedness. We evaluated both semantic relatedness and position relatedness on Textbook dataset in iPRL, and the result is shown in Table 5. From the table, we can see that (i) Both semantic relatedness and position relatedness achieve a better performance. (ii) Position relatedness outperforms semantic relatedness

\begin{tabular}{|c|c|c|c|}
\hline TextRank & THUCKE & AutoPhrase & DsCE \\
\hline \multicolumn{4}{|c|}{ Calculus } \\
\hline $\begin{array}{l}\text { 函数 } \\
\text { function }\end{array}$ & $\begin{array}{l}\text { 函数 } \\
\text { function }\end{array}$ & $\begin{array}{c}\text { 最小正周期 } \\
\text { minimal positive period }\end{array}$ & $\begin{array}{c}\text { 微分中值定理 } \\
\text { differential mean } \\
\text { value theorem }\end{array}$ \\
\hline $\begin{array}{l}\text { 方程 } \\
\text { function }\end{array}$ & $\begin{array}{l}\text { 极限 } \\
\text { limit }\end{array}$ & $\begin{array}{c}\text { 常系数线性微分方程 } \\
\text { linear differential equation } \\
\text { with constant coefficients }\end{array}$ & $\begin{array}{c}\text { 柯西中值定理 } \\
\text { Cauchy mean value } \\
\text { theorem }\end{array}$ \\
\hline $\begin{array}{l}\text { 级数 } \\
\text { series }\end{array}$ & $\begin{array}{l}\text { 方程 } \\
\text { function }\end{array}$ & $\begin{array}{c}\text { 分部积分法 } \\
\text { integration by parts }\end{array}$ & $\begin{array}{c}\text { 微分公式 } \\
\text { differential formula }\end{array}$ \\
\hline \multicolumn{4}{|c|}{ Data Structure } \\
\hline $\begin{array}{c}\text { 算法 } \\
\text { algorithm }\end{array}$ & $\begin{array}{l}\text { 数据 } \\
\text { data }\end{array}$ & $\begin{array}{c}\text { 索引顺序文件 } \\
\text { indexed sequential file }\end{array}$ & $\begin{array}{c}\text { 线性链表 } \\
\text { linear linked list }\end{array}$ \\
\hline $\begin{array}{c}\text { 节点 } \\
\text { node }\end{array}$ & $\begin{array}{l}\text { 存储 } \\
\text { storage }\end{array}$ & $\begin{array}{c}\text { 数组元素 } \\
\text { array element }\end{array}$ & $\begin{array}{l}\text { 数组下标 } \\
\text { array index }\end{array}$ \\
\hline $\begin{array}{l}\text { 查找 } \\
\text { search }\end{array}$ & $\begin{array}{c}\text { 算法 } \\
\text { algorithm }\end{array}$ & $\begin{array}{c}\text { 空指针 } \\
\text { null pointer }\end{array}$ & $\begin{array}{c}\text { 指针变量 } \\
\text { pointer variable }\end{array}$ \\
\hline \multicolumn{4}{|c|}{ Physics } \\
\hline $\begin{array}{c}\text { 运动 } \\
\text { movement }\end{array}$ & $\begin{array}{c}\text { 运动 } \\
\text { movement }\end{array}$ & $\begin{array}{c}\text { 迈克耳孙干涉仪 } \\
\text { michelson interferometer }\end{array}$ & $\begin{array}{c}\text { 电场强度 } \\
\text { electric field strength }\end{array}$ \\
\hline $\begin{array}{l}\text { 物体 } \\
\text { object }\end{array}$ & $\begin{array}{c}\text { 速度 } \\
\text { velocity }\end{array}$ & $\begin{array}{c}\text { 质心运动定理 } \\
\text { theorem of the motion for } \\
\text { center of mass }\end{array}$ & $\begin{array}{c}\text { 涡旋电场 } \\
\text { vortex electric field }\end{array}$ \\
\hline $\begin{array}{c}\text { 速度 } \\
\text { velocity }\end{array}$ & $\begin{array}{c}\text { 过程 } \\
\text { procedure }\end{array}$ & $\begin{array}{l}\text { 尼科耳棱镜 } \\
\text { Nicol prism }\end{array}$ & $\begin{array}{l}\text { 电场能量 } \\
\text { field energy }\end{array}$ \\
\hline
\end{tabular}

Figure 6: Examples of domain-specific concepts extracted for each domain with different methods, where the English phrases under the Chinese phrases are the translations.

slightly. The position of concepts may be more suitable for deducing prerequisite relations.

(4) Evaluation with iterative learning. Figure 7 shows the performance of $\mathbf{i P R L} \mathbf{L}^{p}$ on Textbook dataset varies with the iterations. The interaction between Learning based Model and Recovery based Model in iPRL can really make the performance better gradually.

Finally, we shows some examples of prerequisite relations among concepts extracted by iPRL in Figure 8 .

\section{Related Work}

\section{Domain-specific Concept Extraction}

Keyword extraction is the most related task to domainspecific concept extraction. There are several approaches to extract keywords. Supervised learning methods usually extract syntactic and lexical features for keyword extraction. For example, KEA (Witten et al. 1999) proposed two features: TFIDF and first occurrence, and then used a clas- 


\begin{tabular}{c|c|c|c|c}
\hline Method & measure & CAL & DS & PHY \\
\hline \multirow{3}{*}{ iPRL } & $P$ & 91.38 & 58.86 & 71.69 \\
\cline { 2 - 5 } & $R$ & 55.58 & 64.80 & 57.85 \\
\cline { 2 - 5 } & $F_{1}$ & 69.12 & 61.69 & 64.06 \\
\hline \multirow{3}{*}{ iPRL $^{s}$} & $P$ & 92.96 & 59.16 & 69.70 \\
\cline { 2 - 5 } & $R$ & 54.21 & 66.14 & 60.09 \\
\cline { 2 - 5 } & $F_{1}$ & 68.48 & 62.46 & 64.54 \\
\hline \multirow{3}{*}{ iPRL $^{p}$} & $P$ & 92.48 & 60.16 & 71.12 \\
\cline { 2 - 5 } & $R$ & 56.03 & 65.25 & 59.61 \\
\cline { 2 - 5 } & $F_{1}$ & $\mathbf{6 9 . 7 8}$ & $\mathbf{6 2 . 6 0}$ & $\mathbf{6 4 . 8 6}$ \\
\hline
\end{tabular}

Table 5: Performance of concept relatednesss(\%) on Textbook dataset, where ${ }^{s}$ and ${ }^{p}$ denote the semantic relatedness and position relatedness

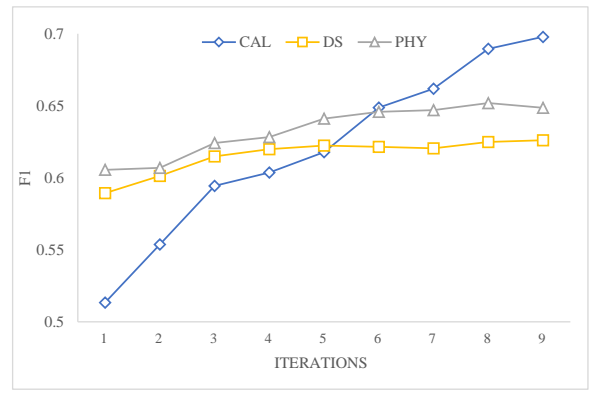

Figure 7: Performance of $\mathrm{iPRL}^{p}$ on Textbook varies with the iterations

sifier to determine the keywords. Recently, an encoderdecoder framework (Meng et al. 2017) was applied to generate keyphrase. Unsupervised learning methods usually apply graph-based semantic relatedness measures for keyword extraction. For example, TextRank (Mihalcea and Tarau 2004) represented a document as a term graph, and used a graphbased ranking algorithm to assign importance scores to terms. Tixier, Malliaros, and Vazirgiannis (2016) proposed a graph degeneracy-based approach for keyword extraction.

However, these researches mainly focused on the selection of words that can best describe the document, but ignore the fact that domain-specific concepts are usually complex and fine-grained.

Recently, several works (Liu et al. 2015; Shang et al. 2018) focus on how to extract high-quality phrases. SegPhrase (Liu et al. 2015) proposed a segmentationintegrated framework by integrating phrase extraction and phrasal segmentation to mutually benefit each other. AutoPhrase (Shang et al. 2018) extended SegPhrase by introducing two new technologies: robust positive-only distant training, and POS-guided phrasal segmentation.

\section{Prerequisite Relation Learning}

A few efforts aim to learn prerequisite relations from educational data, such as courses (Yang et al. 2015; Liang et al. 2015; Liu et al. 2016; Liang et al. 2017), MOOCs (Pan et al. 2017a), textbooks (Wang et al. 2016; Liang et al. 2018) and scientific papers(Gordon et al. 2016).

The statistical information such as reference distance and cross-entropy (Liang et al. 2015; Gordon et al. 2016) was

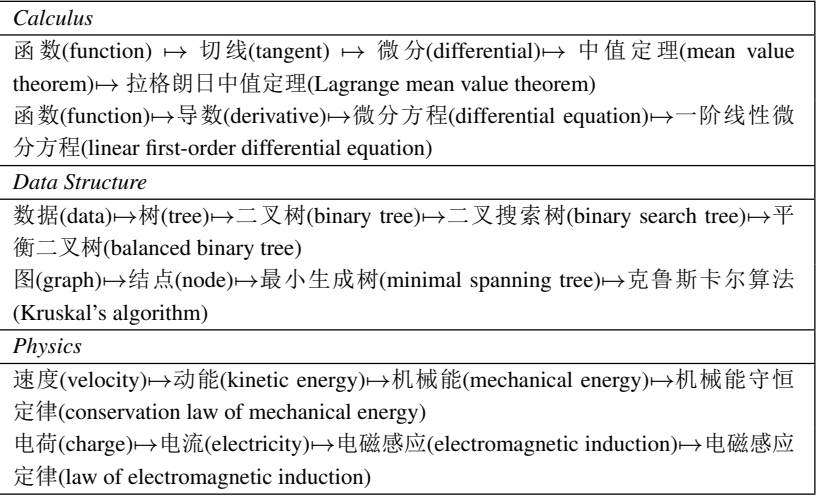

Figure 8: Examples of prerequisite relations extracted by iPRL.

proposed to measure the prerequisite relations among concepts in courses or scientific papers. While Pan et al. (2017a) proposed semantic, contextual and structural features to detect prerequisite relations among concepts in MOOCs.

Other works utilized the dependency among courses or textbooks. For example, (Yang et al. 2015; Liu et al. 2016) proposed a concept graph learning framework for withinand cross-level inference of prerequisite relations at the course-level and the concept-level directed graphs. (Liang et al. 2017) addressed the problem of recovering concept prerequisite relations from university course dependencies. (Wang et al. 2016) proposed a joint optimization model for concept map extraction from textbooks that utilizes the mutual interdependency between concept extraction and prerequisite relation learning. They further applied active learning to the concept prerequisite learning (Liang et al. 2018).

\section{Conclusion}

In this paper, we propose a domain-specific concept extraction approach and an iterative prerequisite relation learning approach. This approach can extract domain-specific concepts and learn the prerequisite relations without human labeled data. In experiments, we evaluated our approach on two real-world datasets Textbook Dataset and MOOC Dataset, and validated that our approach can achieve better performance than existing methods. In addition, we also illustrated some examples of the results of our approach. In future, we plan to use deep learning and active learning technologies to further improve the performance.

\section{Acknowledgments}

This work is supported by the Zhejiang Provincial Natural Science Foundation of China (No. LY17F020015), the Fundamental Research Funds for the Central Universities (No. 2017FZA5016), CKCEST, and MOE-Engineering Research Center of Digital Library.

\section{References}

Falmagne, J.-C.; Cosyn, E.; Doignon, J.-P.; and Thiéry, N. 2006. The assessment of knowledge, in theory and in prac- 
tice. In Missaoui, R., and Schmidt, J., eds., Formal Concept Analysis, 61-79. Berlin, Heidelberg: Springer Berlin Heidelberg.

Finch, G. 2000. Linguistic Terms and Concepts. How to study. Macmillan.

Gordon, J.; Zhu, L.; Galstyan, A.; Natarajan, P.; and Burns, G. 2016. Modeling concept dependencies in a scientific corpus. In Proceedings of the 54th Annual Meeting of the Association for Computational Linguistics (Volume 1: Long Papers), volume 1, 866-875.

Liang, C.; Wu, Z.; Huang, W.; and Giles, C. L. 2015. Measuring prerequisite relations among concepts. In Proceedings of the 2015 Conference on Empirical Methods in Natural Language Processing, 1668-1674.

Liang, C.; Ye, J.; Wu, Z.; Pursel, B.; and Giles, C. L. 2017. Recovering concept prerequisite relations from university course dependencies. In AAAI, 4786-4791.

Liang, C.; Ye, J.; Wang, S.; Pursel, B.; and Giles, C. L. 2018. Investigating active learning for concept prerequisite learning. Proc. EAAI.

Liu, J.; Shang, J.; Wang, C.; Ren, X.; and Han, J. 2015. Mining quality phrases from massive text corpora. Proceedings. ACM-Sigmod International Conference on Management of Data 2015:1729-1744.

Liu, H.; Ma, W.; Yang, Y.; and Carbonell, J. 2016. Learning concept graphs from online educational data. Journal of Artificial Intelligence Research 55:1059-1090.

Liu, Z.; Chen, X.; and Sun, M. 2011. A simple word trigger method for social tag suggestion. In EMNLP.

Margolis, E., and Laurence, S. 1999. Concepts: core readings. Mit Press.

Meng, R.; Zhao, S.; Han, S.; He, D.; Brusilovsky, P.; and Chi, Y. 2017. Deep keyphrase generation. In ACL.

Mihalcea, R., and Tarau, P. 2004. Textrank: Bringing order into texts.

Mikolov, T.; Sutskever, I.; Chen, K.; Corrado, G. S.; and Dean, J. 2013a. Distributed representations of words and phrases and their compositionality. In Advances in neural information processing systems, 3111-3119.

Mikolov, T.; Sutskever, I.; Chen, K.; Corrado, G. S.; and Dean, J. 2013b. Distributed representations of words and phrases and their compositionality. In NIPS.

Pan, L.; Li, C.; Li, J.; and Tang, J. 2017a. Prerequisite relation learning for concepts in moocs. In Proceedings of the 55th Annual Meeting of the Association for Computational Linguistics (Volume 1: Long Papers), volume 1, 1447-1456.

Pan, L.; Wang, X.; Li, C.; Li, J.-Z.; and Tang, J. 2017b. Course concept extraction in moocs via embedding-based graph propagation. In IJCNLP.

Shang, J.; Liu, J.; Jiang, M.; Ren, X.; Voss, C. R.; and Han, J. 2018. Automated phrase mining from massive text corpora. IEEE Transactions on Knowledge and Data Engineering $1-1$.

Talukdar, P. P., and Cohen, W. W. 2012. Crowdsourced comprehension: predicting prerequisite structure in wikipedia. In
Proceedings of the Seventh Workshop on Building Educational Applications Using NLP, 307-315. Association for Computational Linguistics.

Tixier, A. J.-P.; Malliaros, F. D.; and Vazirgiannis, M. 2016. A graph degeneracy-based approach to keyword extraction. In EMNLP.

Wang, S., and Liu, L. 2016. Prerequisite concept maps extraction for automatic assessment. In Proceedings of the 25th International Conference Companion on World Wide Web, 519-521. International World Wide Web Conferences Steering Committee.

Wang, S.; Ororbia, A.; Wu, Z.; Williams, K.; Liang, C.; Pursel, B.; and Giles, C. L. 2016. Using prerequisites to extract concept maps from textbooks. In Proceedings of the 25 th acm international on conference on information and knowledge management, 317-326. ACM.

Witten, I. H.; Paynter, G. W.; Frank, E.; Gutwin, C.; and Nevill-Manning, C. G. 1999. Kea: Practical automatic keyphrase extraction. In $A C M D L$.

Yang, Y.; Liu, H.; Carbonell, J.; and Ma, W. 2015. Concept graph learning from educational data. In Proceedings of the Eighth ACM International Conference on Web Search and Data Mining, 159-168. ACM. 